\title{
论 文
}

\section{一种基于轮廓和尺度不变特征的环境星影像 全自动配准方法}

倪希亮 ${ }^{(12)}$, 曹春香 ${ }^{(1)}$, 丁琳 ${ }^{(3)}$, 江涛 ${ }^{(4)}$, 张影 ${ }^{(1)}$, 贾慧聪 ${ }^{(1)}$, 李光赫 ${ }^{(2)}$, 赵坚 ${ }^{(1)(2)}$ 陈伟 ${ }^{(1)}$ ，姬伟 ${ }^{(1)}$ ，徐敏 ${ }^{(1)}$ ，高孟绪 ${ }^{(1)}$ ，郑盛 ${ }^{(1) 2}$ ，田蓉 ${ }^{(1)}$ ，刘诚 ${ }^{(1)}$ ，李莎 ${ }^{(1)}$

(1) 中国科学院遥感应用研究所遥感科学国家重点实验室, 北京 100101 ;

(2) 中国科学院研究生院, 北京 100049;

(3) 中国科学院遥感应用研究所, 北京 100101 ;

(4) 山东科技大学测绘学院, 青岛 266510;

(5) 北京工业大学艺术设计学院, 北京 100124

* 联系人, E-mail: cao413@irsa. ac. cn

摘要由于环境星 CCD 影像和红外影像之间存在很大的亮度、分辨率以及视角差异, 同时 具有局部扭曲现象, 实现二者全自动配准非常困难. 针对以上难题, 本文提出了一种基于轮 遊和尺度不变特征的全自动配准方法. 首先, 利用轮廓特征配准算法实现粗配准, 得到基准 影像和待配准影像大致配准区域; 然后, 利用局部自适应的尺度不变特征配准算法实现影像 的精配准。影像分块方法被引入到了精配准的过程中, 不仅使得精配准得到的配准点对在影 像中均匀分布, 而且能够进一步提高待配准影像的校正精度. 针对环境星影像做了大量配准

关键词 自动配准 轮廊 尺度不变特征 粗配准 精配准 局部自适应 实验, 结果表明了本文提出的基于轮廓和尺度不变特征配准算法的有效性和精确性.

环境与灾害小卫星星座 $(\mathrm{HJ}-1 \mathrm{~A} / \mathrm{B})$ 可以实现环境 污染和自然灾害的大范围监测, 可以分析生态环境 变化的趋势, 有利于抑制生态恶化和环境污染, 有助 于提高环境监管与保护, 减小城市灾害的潜在风 险 ${ }^{[1 ~ 6]}$. 环境星有着大视角和宽轨道几何成像特征, 尽管卫星已经进行了系统校正, 但是环境星影像仍 然具有大范围的几何扭曲现象 ${ }^{[7]}$. 为了给环境星影像 遥感应用提供更多的技术支持，快速全自动的几何 配准是非常必要的.

\section{1 配准技术的发展}

随着遥感技术的快速发展，过去 20 年间遥感影 像的配准技术已经有了巨大的进步. Strunz ${ }^{[8]}$ 提出了 一种自动检测控制点位置的技术流程，应用于遥感 影像配准中产生了很好的效果. Dai 等 ${ }^{[9]}$ 提出了一种 基于轮廓特征的配准算法, 也能够实现遥感影像较 高精度的配准. Lowe $\mathrm{e}^{[10,11]}$ 提出了一种尺度不变特征 配准算法, 为具有不同分辨率遥感影像的几何配准 提供了重要的支持. 但是, 以上算法在环境星影像的

英文引用格式: Ni X L, Cao C X, Ding L, et al. A fully automatic registration approach based on contour and SIFT for HJ-1 images. Sci China Earth Sci, 2012, doi: 10.1007/s11430-012-4455-7 
配准过程中都会出现较为严重的错配问题.

在遥感影像配准过程中, 为了更好的提高配准 的精确性和有效性, 基准影像和待配准影像的大致 配准区首先需要确定. 通常情况下有两种方法: 一种 是基于地理坐标,一种是人工的选取三个配准点. 当 基准影像和待配准影像的地理坐标缺失时, 以上两 种方法就无法实现全自动配准的要求. 因此, 自动检 测影像的大致配准区域是非常必要的. 基于轮廓的 配准算法可以快速自动获取两幅影像的粗配准范围 并且具有很高的精度.

当粗配准区域确定之后, 以往的方法对于来自 于同一传感器相同分辨率的遥感影像配准也可以达 到很高的精度. 但对于不同分辨率、不同视角、不同 传感器多源遥感影像的配准往往很难达到理想的配 准效果, 例如, $\mathrm{HJ}-1 \mathrm{~A} / \mathrm{B}$ 的配准以及其光学影像和红 外影像的配准. 在这种情况下, 尺度不变特征配准算 法由于其对于不同尺度、灰度以及视角变化等保持不 变的特性被引入到本文提出的配准算法当中.

然而, 原始的尺度不变特征算法仍然具有错配 和时间复杂度高的缺点, 如果直接应用于遥感影像 配准就不能达到预期的效果 ${ }^{[12]}$. 尤其对于多源遥感 影像, Kennedy 等 ${ }^{[13]}$ 和 Bentoutou 等 ${ }^{[14]}$ 表明, 原始的尺 度不变特征算法对于复杂成像条件的遥感影像很难 实现特征点一一对应配准, 致使产生很严重的错配 现象. 因此, 针对上述问题, 本文提出了一种基于轮 廓和尺度不变特征的全自动配准新方法.

\section{2 算法流程}

针对多源遥感影像的特点, 本文提出了一种全 自动配准算法. 给定基准影像和待配准影像, 利用本 文算法即可实现其自动配准, 配准流程如图 1 所示. 首先, 执行基准影像和待配准影像的初配准, 利用轮 廓特征检测算法得到基准影像和待配准影像的大致 配准区域, 将大致配准区域作为新的基准影像和待 配准影像; 然后对大致配准区域进行均匀分块, 获取 影像块序列; 第三步, 在相应的基准影像和待配准影 像块序列中分别检测得到具有相同数量的尺度不变 特征点及其特征描述; 第四步, 通过局部自适应的方 法实现相应影像块的精配准; 第五步, 一致性检测方 法去除错配点进一步提高配准的精度; 最后, 利用成 熟的校正算法，例如多项式以及分段线性转换技术

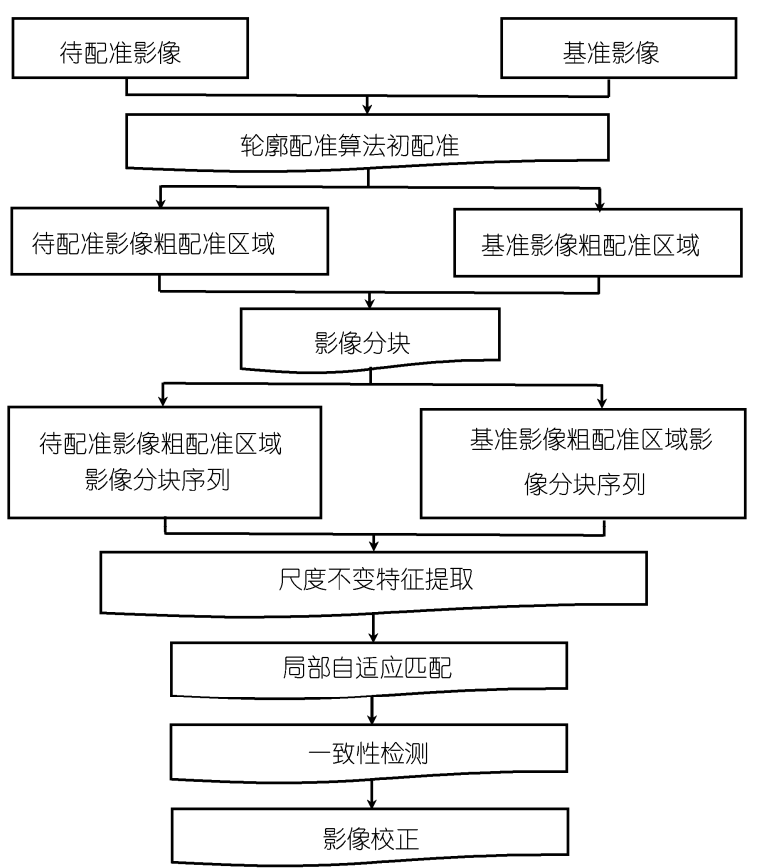

图 1 算法整体流程图

等 ${ }^{[15]}$ 算法实现待配准影像的校正. 下面针对本文算 法的每一步都给出了详细的解释. 同时, 通过三组不 同特征的环境星影像的配准实验具体说明本文算法 的有效性. 这三组实验分别是: HJ-1A/B CCD 影像 的配准, HJ-1A CCD 影像与 HJ-1B IRS 影像的配准 以及 HJ-1B CDD 影像和 CBERS 02 CCD 影像的配 准, 所有实验影像均为不同时相. 实验结果表明, 本 文算法可以实现环境星影像之间以及环境星与其他 传感器影像等多源遥感影像的高精度、全自动配准.

\section{3 基于轮廓特征的初配准}

初配准过程采用了轮廓特征方法确定基准影像 和待配准影像之间的粗配准区域. 轮廓特征配准算 法的流程图如图 2 所示, 此过程可分为五个关键步骤: 第一步，首先应用高斯拉普拉斯函数滤波，然后利用 零值交叉变换得到轮廓特征; 第二步, 选取闭轮廓进 行编码; 第三步，利用不变矩特征实现轮廓匹 配 ${ }^{[16 ~ 18] ;}$; 第四步，通过一致性检测算法去除错配轮 廓 ${ }^{[19]}$; 最后, 获取基准影像和待配准影像的粗配准 区域.

当然，本文提出的粗配准过程需要一个先决条 件：基准影像和待配准影像应当具有相对丰富的纹 


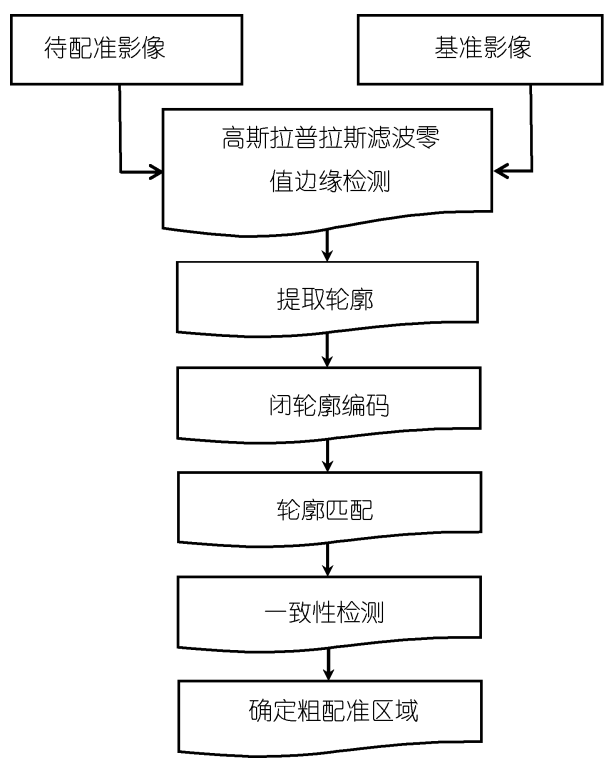

图 2 轮廓特征配准算法流程图

理特征; 这主要是因为当影像缺乏纹理特征时轮廓 配准算法无法获取足够的轮廓特征实现错配准. 但 由于环境星影像具有大幅宽和纹理较为丰富的特征, 因此通过轮廓特征匹配算法获取粗配准区域是可行 的.

\section{4 影像分块处理}

考虑到遥感影像的大数据量以及严重的错配问 题, 对影像进行分块处理是非常必要的. 此过程对基 准影像和待配准影像进行分块, 需要确保相似区域 的影响块具有相同的序号, 记作 $\left\{A_{i}\right\},\left\{B_{i}\right\}$. 假设基准 影像大小为 $M \times N$, 待配准影像大小为 $P \times Q$, 它们被分 割为 $m \times n$ 块. 最终, 基准影像的影像块大小为 $(M / m) \times$ $(N / n)$, 待配准影像的影像块大小为 $(P / m) \times(Q / n)$.

\section{5 尺度不变特征点提取}

尺度不变特征算法基于图像特征尺度选择的思 想建立图像的多尺度空间，在不同尺度下检测到同 一个特征点, 确定特征点位置的同时确定其所在尺 度, 以达到尺度不变目的. 该过程主要分为三步进行: 尺度空间的建立、关键点检测以及关键点描述. 在本 文中, 在每一个影像块中都提取了大量的特征点和 特征算子. 最终, 在同一个影像块中提取的特征描述
算子组成特征描述算子集合. 例如, $A_{1}$ 是基准影像的 第一个影像块; $B_{1}$ 是待配准影像的第一个影像块. 从 $A_{1}$ 中提取的描述算子组成集合 $\left\{f_{j}\right\}_{1}$,从 $B_{1}$ 中提取的 描述算子组成集合 $\left\{g_{j}\right\}_{1}$.

\section{6 局部自适应匹配}

\section{1 最优欧氏距离匹配}

本文算法利用了局部搜索和几何约束理论对特 征描述算子进行匹配. 假设 $\left\{A_{i}\right\}$ 和 $\left\{B_{i}\right\}$ 是两个影像子 块, $T_{A_{i}}=\left\{f_{j}\right\}_{i}$ 是子影像块 $A_{i}$ 的特征向量集, $T_{B_{i}}=\left\{g_{j}\right\}_{i}$ 是 子影像块 $B_{i}$ 的特征向量集. 在上面的等式中, $i$ 是子 影像块的序号, $j$ 是子影像块中关键点的序号.

首先确定基准影像子块每一个特征点的目标搜 索区域. 影像子块 $A_{i}$ 中的特征点目标搜索区域是与 之相对应的影像子块 $B_{i}$. 然后, 计算 $A_{i}$ 中的特征向量 与 $B_{i}$ 中的特征向量的欧式距离. 选出最近邻特征点 和次近邻特征点的距离, 然后计算最近邻特征点和 次近邻特征点的距离比值. 假设 $d i s_{j 1}$ 和 $d i s_{j 2}$ 分别是最 近邻和次近邻距离, 它们的比率 $k_{j}$ 为

$$
k_{j}=d i s_{j 1} / d i s_{j 2} \text {. }
$$

最终, 将 $k_{j}$ 从小到大进行排序. 根据需要, 可以 选择最优的前 $n$ 个配准点. 本文仅选择了最小的 $k_{j}$ 对应的配准点对作为最优欧式距离的配准点.

在原始的 SIFT 配准算法中, 配准点的确定也是 根据 $k_{j}$. 但是阈值 $r$ 需要提前确定. 当 $k_{j}<r$, 两个点将 被作为正确的配准点. 显然, 这种方法具有一定的局 限性，因为正确的估算 $r$ 是一个问题. 同时，在多源 遥感影像的配准过程中, 最优的阈值 $r$ 也是不同的. 然而, 本文提出的最优欧式距离匹配的方法就不会 存在如何确定阈值 $r$ 的问题，因为我只选择了一个最 优的 $k_{j}$, 不会因为阈值的选取而产生错配. 因此, 本 文应用的最优欧氏距离匹配算法要比原始的匹配方 法更有优势.

\section{2 一致性检测}

在均匀分块的情况下，每一相同序号影像块的 对应区域是同一区域时，经过改进 SIFT 匹配算法， 配准所得结果一般情况下都是正确的. 然而有时相 同序号影像块的对应区域重叠部分较少，容易引起 错配，此时一致性检验显得就尤为重要 ${ }^{[20]}$. 一致性检 
测的原理是：让 $\overline{A_{1} A_{2}}$ 代表同一幅影像中的点 $A_{1}$ 和点 $A_{2}$ 之间的距离. 对影像 1 和影像 2 中特征点的两个集 合 $\left\{A_{i} \mid i=1,2,3 \cdots\right\}$ 和 $\left\{B_{i} \mid i=1,2,3 \cdots\right\}$, 计算比率为

$$
\overline{A_{i} A_{j}} / \overline{B_{i} B_{j}}, i=1, \cdots m, j=i+1, \cdots m .
$$

这些形成一个簇, 因此, 散落在聚类中心较远的会被 当作错误匹配而拒绝.

\section{7 实验结果}

应用本文提出的方法进行了大量的实验，实验 主要针对来自不同传感器的环境星 CCD 影像以及 $\mathrm{HJ}$ 星 $\mathrm{CCD}$ 影像和 $\mathrm{HJ}$ 星红外影像之间的配准. 同时, HJ-1 影像与 CBERS 02 影像之间的配准也做了大量 实验, 目的是验证本文提出的算法对于多源遥感影 像之间配准的有效性. 因此, 实验分成三类：(1) 不 同时相获取的 HJ-1A CCD 和 HJ-1B CCD 中分辨率 遥感影像之间的配准实验. (2) 中分辨率 HJ-1A CCD 影像与低分辨 HJ-1B IRS 影像配准实验. 它们之间存 在较大的几何变形、分辨率差异以及亮度差异. (3) CBERS 02 多光谱影像与 HJ-1B CCD 影像配准. 由于 来自于不同传感器, 二者之间存在着很大的视角差 异, 同时具有分辨率、亮度差异以及几何变形.

针对以上三个实验, 本文在下面对其进行分析. 研究区域主要集中在天津和河北, 如图 3 所示. 所有 的影像都是 8 位灰度图像, 所有的配准实验都是在 $2.9 \mathrm{GHz}$ 的计算机上进行.

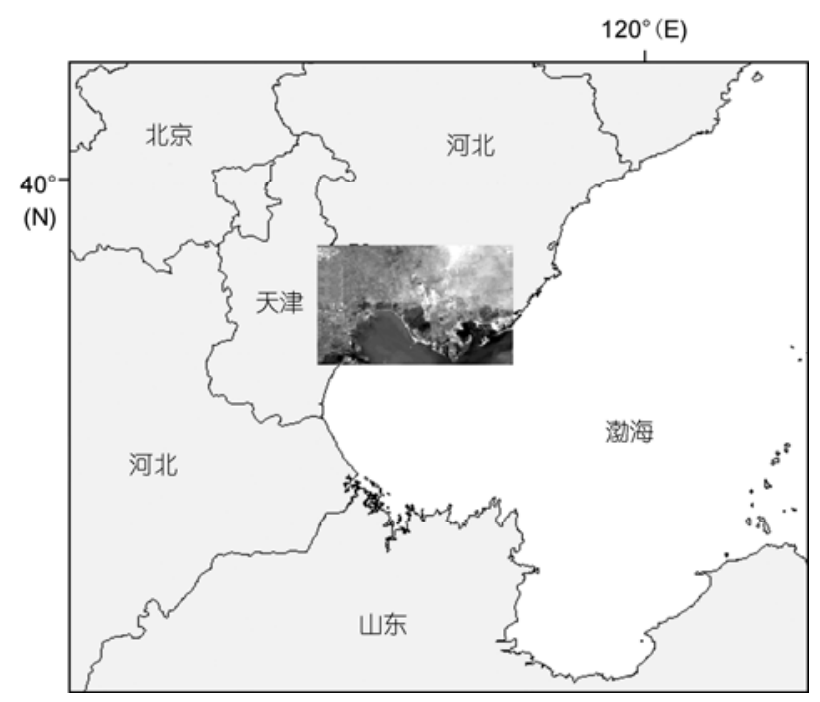

图 3 研究区所在位置

\section{1 实验一中分辨率 HJ-1A CCD 影像与 HJ-2 CCD 影像配准}

本实验使用 HJ-1 A CCD 影像作为基准影像, 获 取时间为 2009 年, 影像大小 $3894 \times 6896$, 空间分辨率 为 $30 \mathrm{~m}$, 如图 4(a)所示; HJ-1B CCD 影像作为待配准 影像，获取时间 2010 年，影像大小 $4230 \times 6652$, 空间 分辨率为 $30 \mathrm{~m}$, 如图 4(b)所示. 由于来自于不同的卫 星传感器, 两幅影像之间存在旋转、亮度差异以及几 何变形. 利用以往的配准算法实现其高精度的自动 配准有一定困难. 利用本文提出的算法就可以达到 很高的配准结果.

在预处理阶段, 分别从 $\mathrm{HJ}-1 \mathrm{~A}$ CCD 影像和 HJ-1B CDD 影像中提取相应的轮廓特征, 如图 5 所 示. 基于本文第 3 节的理论, 得到 6 个配准点. 通过 六个配准点得到基准影像和待配准影像的粗配准区 域, 作为新的基准影像和待配准影像.

在影像分块分块过程中, 新得到的基准影像和 待配准影像分别均匀分成影像块序列, 影像块数目 为 $m \times n, m=4, n=4$; 所得到的影像块序列分别为 $\left\{A_{1}\right.$, $\left.A_{2}, A_{3}, \cdots A_{16}\right\}$ 和 $\left\{B_{1}, B_{2}, B_{3}, \cdots B_{16}\right\}$. 然后利用尺度不 变特征算法分别从每一个相应的影像块中提取特征 点和特征描述，经过局部自适应匹配和一致性检测 之后, 最终得到 14 个均匀分布的配准点, 如图 6 所示. 所有步骤总共耗时 $267 \mathrm{~s}$. 为了验证配准的精度, 将 14 个均匀分布的配准点输入到 ENVI 软件中, 得到均
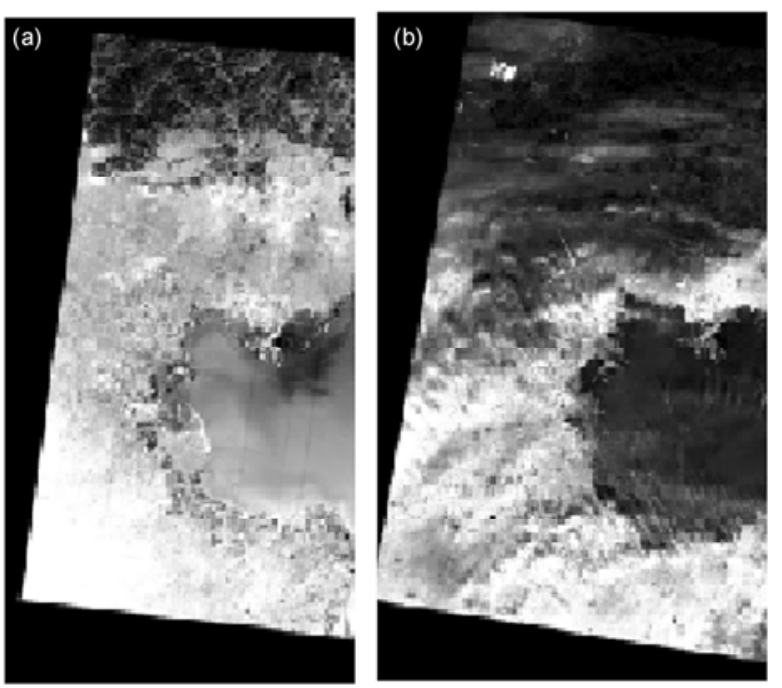

图 4 实验一基准影像和待配准影像

(a) 基准影像(HJ-1A CCD); (b) 待配准影像(HJ-1B CCD)

1248 
方根误差(RMSE)小于 0.35 . 作为比较, 人工选取了 25 控制点均匀分布在影像中, 显示的均方根误差 (RMSE) 为 1.43 .

为了验证本文算法的有效性, 配准结果与原始 的 SIFT 算法进行了对比, 具体如表 1 所示. 原始 SIFT 算法的配准结果如图 7 所示. 从图中可以看出, 有非常多的错配产生. 尽管匹配的阈值反复调整, 最 终得到的配准结果仍然有着大量错配. 同时, 大数据 量影像配准经常会使计算机内存溢出. 本文算法由 于采用了分块方法, 因此不会有计算机内存溢出情 况发生.

7.2 实验二中分辨率 $\mathrm{HJ}-1 \mathrm{~A}$ 可见光影像和低分辨 率 HJ-1B 红外影像的配准

本实验对 HJ-1A 可见光影像和低分辨率 HJ-1B 红外影像进行了配准. 其中, 作为基准影像的可见光 影像获取时间是 2009 年, 影像大小为 $1929 \times 3409$, 空间分辨率为 $30 \mathrm{~m}$, 如图 8(a)所示; 作为待配准影像 的红外影像第一波段获取时间是 2009 年, 它的波段
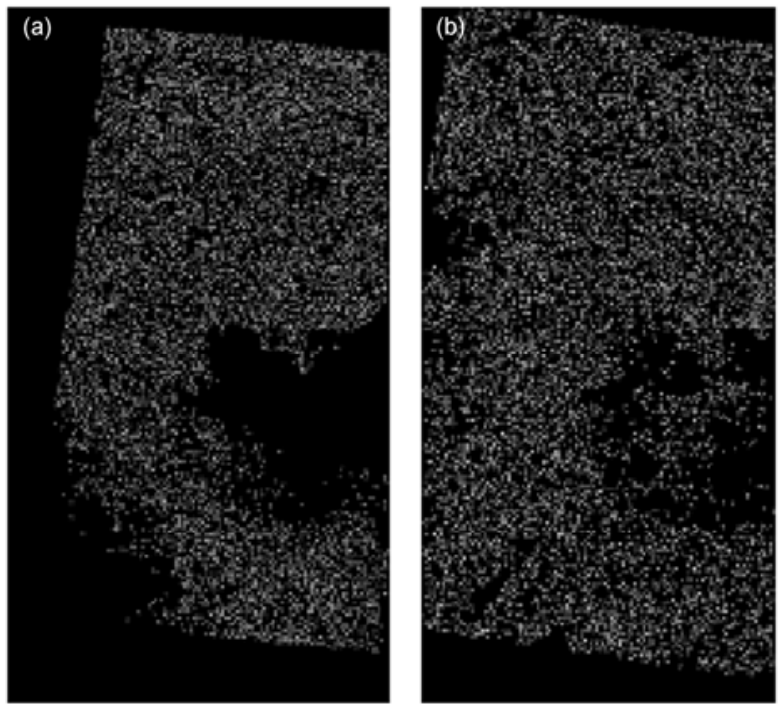

图 5 实验一由基准影像和待配准影像提取的轮廓图 (a) 基准影像轮廓(HJ-1A CCD); (b) 待配准影像轮廓(HJ-1B CCD)
范围是 400 770 nm, 属于近红外波段, 影像大小为 $668 \times 540$ ，空间分辨率为 $150 \mathrm{~m}$ ，如图 8(b)所示.
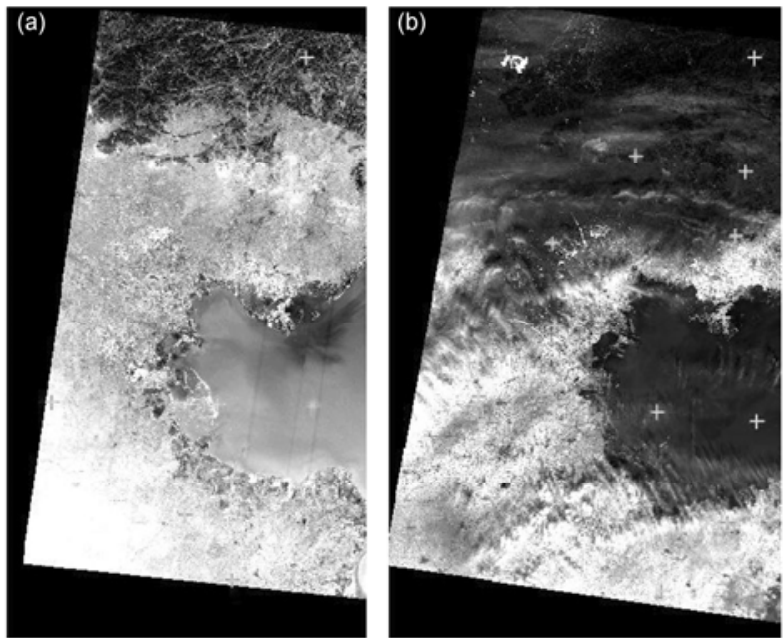

图 6 实验一本文算法配准结果图

(a) 从基准影像中提取的匹配点图(HJ-1A CCD); (b) 从待配准影像 影像中提取到的匹配点图(HJ-1B CCD). 所有配准点均匀分布在基 准影像和待配准影像中

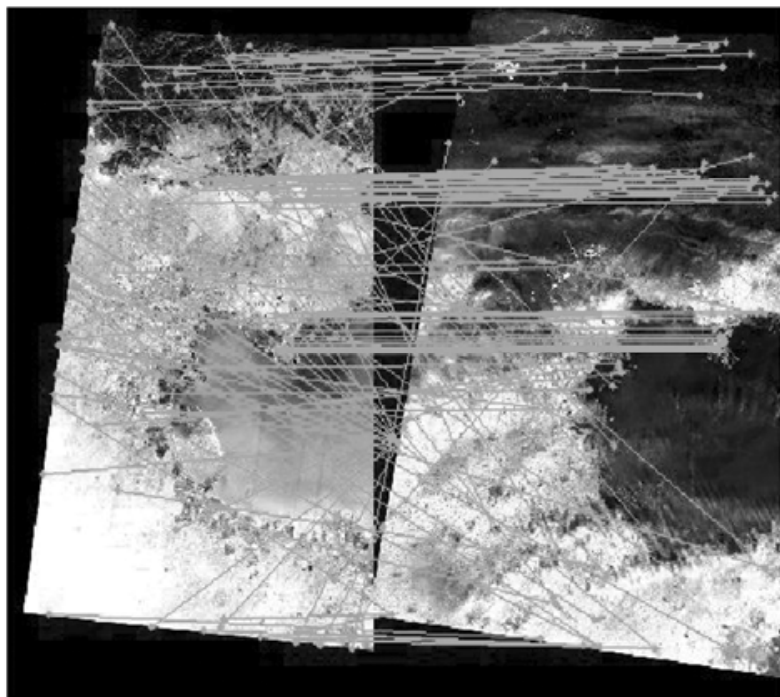

图 7 利用原始的 SIFT 算法 ${ }^{[10]}$ 得到的配准结果图

表 1 实验一本文算法与原始 SIFT 算法的配准结果比较

\begin{tabular}{|c|c|c|c|c|c|c|c|c|}
\hline \multirow{3}{*}{ 本文算法 } & \multicolumn{5}{|c|}{ 时间复杂度(s) } & \multicolumn{3}{|c|}{ 配准精度 } \\
\hline & 初配准 & 分块 & 改进的 SIFT & 一致性检测 & 合计 & 匹配点 & 正确匹配点 & 正确率 \\
\hline & 116 & 1 & 147 & 3 & 267 & 14 & 14 & $100 \%$ \\
\hline \multirow{2}{*}{ 原始 SIFT 算法 } & \multicolumn{5}{|c|}{ 合计 } & 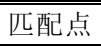 & 正确匹配点 & 正确率 \\
\hline & \multicolumn{5}{|c|}{695} & 766 & 423 & $55 \%$ \\
\hline
\end{tabular}


基准影像和待配准影像之间存在大的分辨率和 灰度差异以及几何变形. 大多情况下由于红外影像 分辨率低, 利用其他配准算法只能提取少量特征点, 从而导致低的配准精度. 本文算法采用了尺度不变 特征技术，可以从低分辨的红外影像中提取大量特 征点, 从而实现与可见光影像的最优配准.

在初配准阶段, 分别从 $\mathrm{HJ}-1 \mathrm{~A}$ CCD 影像和 HJ-1B IRS 影像提取轮廓, 选择最优的三个特征点获 得初配准区域. 这三个特征点是相应三个最优匹配 轮廓的中心. 通过影像分块、精配准以及一致性检测 后, 最终得到 11 个配准点, 如图 9 所示. 为验证配准 结果的正确性，计算了配准点的均方根误差(RMSE)
为 1.18 , 具体的实验结果如表 2 所示.

\section{3 实验三中 CBERS 02 影像和中分辨率 HJ-1B 可见光影像的配准}

本实验选用 CBERS 02 多光谱影像作为基准影 像，影像获取时间为 2007 年 9 月，影像大小为 7380x 6860 , 空间分辨率为 $19.5 \mathrm{~m}$; HJ-1B CCD 作为待配准 影像，影像获取时间为 2011 年 2 月，影像大小为 4177×4409, 空间分辨率为 $30 \mathrm{~m}$. 可见，基准影像和 待配准影像之间存在分辨率差异和视角差异. 利用 本文提出的方法，可以实现二者之间的高精度配准， 最终配准结果如图 10 所示. 从图中可以看出, 三对
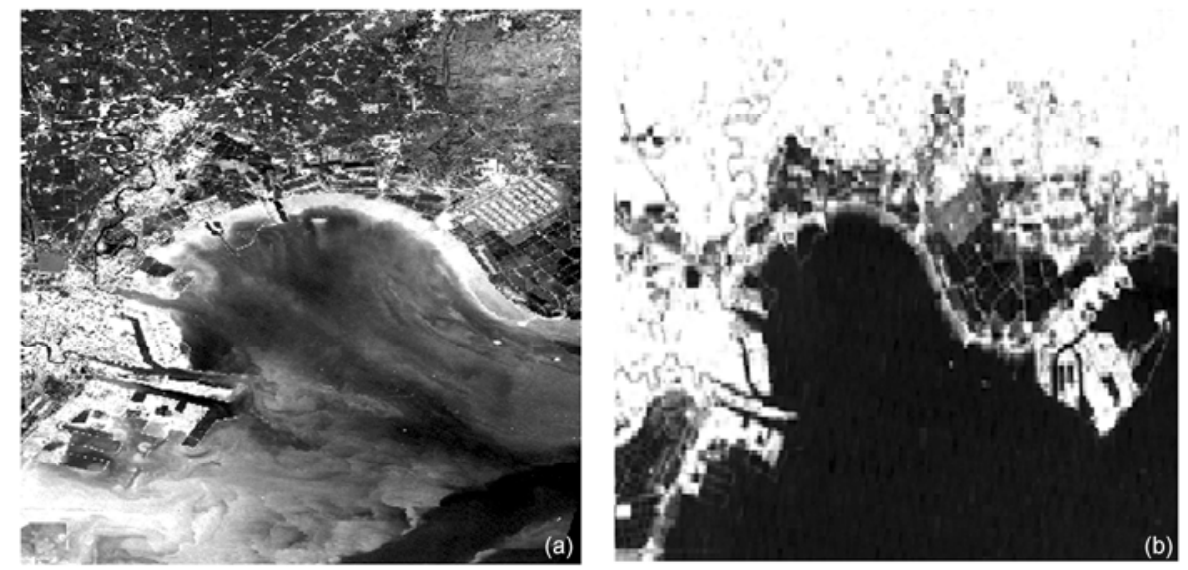

图 8 实验二中基准影像和待配准影像

（a) 基准影像(HJ-1A CCD); (b) 待配准影像(HJ-1B IRS)
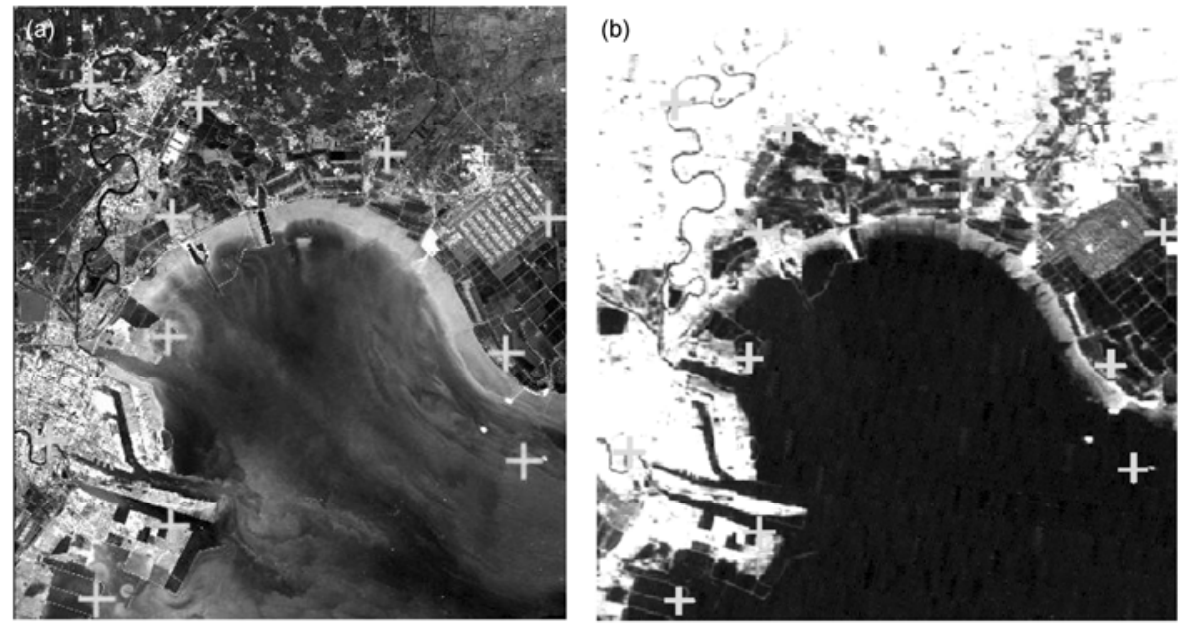

图 9 实验二中本文算法配准结果图

(a) 从基准影像中提取的匹配点图(HJ-1A CCD); (b) 从待配准影像影像中提取到的匹配 0 点图(HJ-1B CCD). 总共 11 对正确的匹配点对 
表 2 实验二本文算法与原始 SIFT 算法的配准结果比较

\begin{tabular}{|c|c|c|c|c|c|c|c|c|}
\hline \multirow{3}{*}{ 本文算法 } & \multicolumn{5}{|c|}{ 时间复杂度(s) } & \multicolumn{3}{|c|}{ 配准精度 } \\
\hline & 初配准 & 分块 & 改进的 SIFT & 一致性检测 & 合计 & 匹配点 & 正确匹配点 & 正确率 \\
\hline & 169 & 1 & 217 & 1 & 387 & 11 & 11 & $100 \%$ \\
\hline \multirow{2}{*}{ 原始 SIFT 算法 } & \multicolumn{5}{|c|}{ 合计 } & 匹配点 & 正确匹配点 & 正确率 \\
\hline & \multicolumn{5}{|c|}{204} & 124 & 32 & $26 \%$ \\
\hline
\end{tabular}

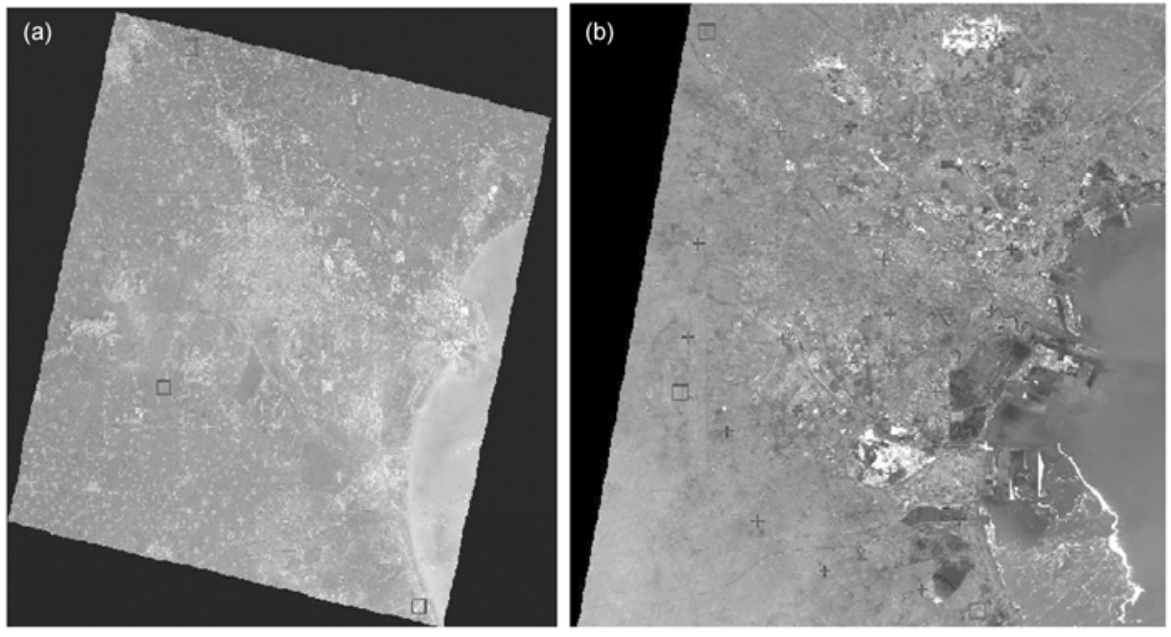

图 10 实验三本文算法配准结果图

(a) 从基准影像(CBERS 02 多光谱影像)提取得到的配准点分布图; (b) 从待配准影像(HJ-1B CCD 影像)提取得到的配准点分布图. 图中的方 框表示由初配准过程中确定的配准轮廓点, 十字交叉表示最终得到的 23 个配准点

表 3 实验三本文算法与原始 SIFT 算法的配准结果比较

\begin{tabular}{|c|c|c|c|c|c|c|c|c|}
\hline \multirow{3}{*}{ 本文算法 } & \multicolumn{5}{|c|}{ 时间复杂度(s) } & \multicolumn{3}{|c|}{ 配准精度 } \\
\hline & 初配准 & 分块 & 改进的 SIFT & 一致性检测 & 合计 & 匹配点 & 正确匹配点 & 正确率 \\
\hline & 169 & 1 & 217 & 4 & 391 & 22 & 22 & $100 \%$ \\
\hline \multirow{2}{*}{ 原始 SIFT 算法 } & \multicolumn{5}{|c|}{ 合计 } & “匹配点 & 正确匹配点 & 正确率 \\
\hline & \multicolumn{5}{|c|}{1034} & 1024 & 321 & $31 \%$ \\
\hline
\end{tabular}

红色标记的配准点确定了两幅影像的粗配准区域; 对粗配准区域的影像分成 $5 \times 5$, 经过尺度不变特征检 测、局部自适应匹配以及一致性检测之后, 最终获得 了 23 对正确的匹配点. 为了进一步验证配准结果的 准确性, 计算得到均方根误差 (RMSE) 为 1.3 , 具体的 实验结果如表 3 所示.

为进一步验证本文算法的有效性, 针对多源遥 感影像进行了大量的配准实验. 大部分实验结果表 明, 本文算法能够得到高精度的配准结果, 并且实现 全自动的影像配准. 然而, 一些特殊情况下, 本文算 法也无法获取高精度的配准结果. 这主要是因为缺 乏纹理的影像以及分辨率差异过大的影像之间(分辨 率相差 8 倍)无法获得轮廓特征确定粗配准区域或者
在初配准区域无法获得足够多的配准点. 尽管如此, 本文算法仍然具有很高的实用价值.

\section{8 结论}

本文提出了一种全自动配准算法, 可以实现 HJ-1 星影像以及其他多源遥感影像的高精度配准. 算法集成了多种不同技术, 包括轮廓配准、尺度不变 特征算法、分块理论、几部自适应策略以及一致性检 测算法. 算法可以在无需人工干预的情况下实现多 源遥感影像的全自动配准. 同时, 配准过程中提取的 匹配点能够均匀分布在配准影像中, 从而提高了影 像校正的精度. 当然, 本文算法还有一些改进之处, 
例如对于缺乏纹理影像的配准问题, 这将是以后的研究重点.

致谢感谢审稿专家提出的宝贵意见.

\section{参考文献}

1 朱海涌. 环境与灾害监测预报小卫星数据应用评价. 干旱环境监测, 2010, 24: 39-42

2 Wang Q, Wu C Q, Li Q, et al. Chinese HJ-1A/B satellites and data characteristics. Sci China Earth Sci, 2010, 53(Suppl. I): 51-57

3 Yang J, Gong P, Zhou J X, et al. Detection of the urban heat island in Beijing using HJ-1B satellite imagery. Sci China Earth Sci, 2010, 53(Suppl. I): 67-73

4 Guo Z F, Chi H, Sun G Q. Estimating forest aboveground biomass using HJ-1 Satellite CCD and ICESat GLAS waveform data. Sci China Earth Sci, 2010, 53(Suppl. I): 16-25

5 Chen W, Cao C X, He Q S, et al. Quantitative estimation of the shrub canopy LAI from atmosphere-corrected HJ-1 CCD data in Mu Us Sandland. Sci China Earth Sci, 2010, 53(Suppl. I): 26-33

6 Sun L, Sun C K, Liu Q H, et al. Aerosol optical depth retrieval by HJ-1/CCD supported by MODIS surface reflectance data. Sci China Earth Sci, 2010, 53(Suppl. I): 74-80

7 Hu C M, Tang P. HJ-1A/B CCD IMAGERY geometric distortions and precise geometric correction accuracy analysis. Geoscience and Remote Sensing Symposium (IGARSS), 2011 IEEE International. July 24-25, 2011. 4050-4053

8 Strunz G. Automation of tie pointing procedure for the geocoding of satellite images. Inter Arch ISPRS, 1994, 30: 793-800

9 Dai X L, Khorram S. A feature-based image registration algorithm using improved chain-code representation combined with invariant moments. IEEE Trans Geosci Remote Sensing, 1999, 37: 2351-2361

10 Lowe D G. Object recognition from local scale-invariant features. Inter Conf Computer Vision, 1999, 37: 2351-2362

11 Lowe D G. Distinctive image features from scale-invariant keypoints. Inter J Computer Vision, 2004, 60: 91-110

12 Gonçalves H, Corte-Real L, Goncalves J A. Automatic image registration through image segmentation and SIFT. IEEE Geosci Remote Sensing Soc, 2011, 49: 2589-2600

13 Kennedy B E, Cohen W B. Auto-matched designation of tie-points for image-image co-registration. Inter J Remote Sensing, 2003, 24 : $3467-3490$

14 Bentoutou Y, Table N, Kpalma K. An Automatic image registration for application in remote sensing. IEEE Trans Geo-sci Remote Sensing, 2005, 43: 2127-2137

15 Brown L G. A survey of image registration techniques. J Acm Comput Surv, 1992, 24: 325-376

16 Li H, Manjunath B S, Mitra S K. A contour based approach to multi-sensor image registration. IEEE Trans Imaging Processing, 1995, 4: 320-334

17 Williams D, Shah M. A fast algorithm for active contours and curvature estimation. CVGIP Image Understanding, 1992, 55: 14-26

18 Haberäcker P. Praxis der Digitalen Bildverarbeitung und Mustererkennung. Hanser Fachbuch, 1995

19 Yue W, Eam K. 2D affine-invariant contour matching using B-spline model. IEEE Trans Pattern Analy Mach Intell, 2007, 29: 1853-1858

20 Canty M J. Image Analysis, Classfication and Change Detection in Remote Sensing. Taylor \& Francls, 2006. 146-147 